\title{
The use of suction blisters to measure sunscreen protection against UVR- induced DNA damage
}

By Gwendal Josse ${ }^{1 *}$, Thierry Douki ${ }^{2}$, Jimmy Le Digabel ${ }^{1}$,Eleonore Gravier ${ }^{1}$ and Emmanuel Questel $^{1}$

1 Centre de Recherche sur la Peau, Pierre Fabre Dermo-Cosmétique, F-31000 Toulouse, France 2 - Univ. Grenoble Alpes, CEA, CNRS, INAC, SyMMES/CIBEST, F-38000 Grenoble

- Corresponding author

Gwendal Josse

Pierre Fabre Dermo-Cosmétique, Centre de Recherche sur la Peau

Hôtel Dieu-Saint Jacques

2 rue Viguerie

31000 Toulouse

Email : gwendal.josse@pierre-fabre.com 


\section{Summary}

Formation of DNA photoproducts caused by solar UV exposure need to be investigated in-vivo and in particular in order to assess sunscreens' level of protection against solar genotoxicity. The study's purposes were: i) to evaluate if the roof of suction blisters are appropriate samples for measuring photoproducts, and ii) to measure in-vivo sunscreen protection against cyclobutane pyrimidine dimers. Skin areas on the interior forearms of eight healthy volunteers were exposed in-vivo to $2 \mathrm{MED}$ of simulated solar radiation (SSR) and to 15 MED on a sunscreen protected area. After irradiation, six suction blisters were induced and the blister roofs were collected. Analysis of SSR-induced CPDs was performed by two independent methods: a chromatography coupled to mass spectroscopy (HPLC-MS/MS) approach and a 3D-imaging of CPD immunostaining by multiphoton microscopy on floating epidermal sheets. HPLC-MS/MS analyses showed that UV-unexposed skin presented no CPD dimers, whereas 2 MED SSR-exposed skin showed a significant number of TT-CPD. The sunscreen covered skin exposed to 15 MED appeared highly protected from DNA damage, as the amount of CPD-dimers remained below the detection limit. The multiphotonimmunostaining analysis consistently showed that no CPD staining was observed on the nonUV-exposed skin. A significant increase of CPD staining intensity and number of CPD-positive cells were observed on the 2 MED SSR-exposed skin. Sunscreen protected skin presented a very low staining intensity and the number of CPD-positive cells remained very close to nonUV-exposed skin. This study showed that suction blister samples are very appropriate for measuring CPD dimers in-vivo, and that sunscreens provide high protection against UVinduced DNA damage. 


\section{Introduction}

UV radiation (UVR) is the most damaging portion of solar light to skin. UVR induces erythema, immunomodulation and is the initiating agent of skin cancers [1,2]. Overexposure to sun has thus to be banned and prevention strategies such as sun avoidance or adequate clothing have been often advertised in prevention campaign. Yet, sun seeking behavior is a strong trend in the general population and use of sunscreen appears as an attractive strategy to limit exposure of skin to UVR. It is thus of major importance to accurately evaluate sun protection products. Today, these are characterized by the sun protection factor (SPF) which measures the ability of a formula to decrease the dose leading to the appearance of an erythema [3]. Yet, erythema is a short term response of inflammatory origin. It does not reflect the protection against long term adverse effects such as skin cancer. Another important point in modern sunscreens is the trend to an increased protection against UVA. Although no standardized method is defined, persistent pigment darkening is often used [4]. Again, this parameter is not related to long term effect of UVR such as skin cancer. The design of new tools for the assessment of efficacy of sunscreens against the carcinogenic effects of sunlight, both its UVB and UVA components, is thus needed. Solar UVR radiation is the main cause of skin cancer because it is an efficient DNA damaging agent. UVB are strongly absorbed by DNA and induce the formation of dimeric lesions between adjacent pyrimidines: cyclobutane pyrimidine dimers (CPDs) and pyrimidine (6-4) pyrimidone photoproducts (64PPs) [5]. These lesions are generally repaired by the excision repair pathway, but when unrepaired can cause mutations (C-> T or CC-> TT), which may lead to tumorogenesis [6]. UVA are much less efficient at damaging DNA. A first well documented pathway involves the photosensitized production of reactive oxygen species that then damage DNA [7]. The resulting lesions are strand breaks and oxidized bases such as 8-oxo-7,8-dihydroguanine. UVA is also absorbed by DNA, although with a much lower efficiency than UVB. UVA excitation of DNA leads to the formation of CPDs [8-11]. UVA-induced 64PPs have been detected by immunological assays $[8,12,13]$ but not by the more specific HPLC-MS/MS technique $[10,11,14]$. Evidence has also been provided for a major role of UVA in conversion of UVBinduced 64PPs into their Dewar valence isomers [15]. Interestingly, CPDs are induced in larger yield than 8-oxoGua in human skin and cells exposed to UVA [10,14,16]. Altogether, pyrimidine dimers appear to be highly relevant DNA damage to the carcinogenic effects of sunlight. 
Accurate determination of the level of pyrimidine dimers in human skin is thus an important issue not only to unravel the biological effects of UVR but also to evaluate photoprotection strategies. A very large body of literature is available on the formation of CPDs and 64PPs in cells. Major pieces of information were also gathered in skin on the depth profile of the formation of photoproducts, the phototype effects, the wavelength effects and the repair kinetics. Much less information is available on photoprotection of DNA in human skin. In-vivo testing is essential because it assures that the tissue response corresponds exactly to real sun exposure conditions. Most available data have been obtained by antibody-based approaches such as immunohistochemistry, immunodot blot or radio immunoassays [17-24]. Data on photoprotection were also obtained on the basis of chromatographic assay [25,26]. The advantage of the latter approach is that results are quantitative and that individual results are provided on each of the possible bipyrimidic photoproducts [27]. A common feature of all these approaches is that they rely on the collection of biopsies in volunteers in in vivo studies. This last point can be a limitation because biopsies are quite invasive and their number for a same donor is limited by ethical considerations.

In order to offer even more precise quantification of photoproducts in skin using a less invasive procedure, we show in the present work that the suction blister, as a skin sampling method, is very appropriate. Two established techniques for photoproduct measurements were applied to this type of skin samples: HPLC-tandem mass spectrometry and immuno-staining. For the latter assay, optimization of the imaging procedure is proposed which makes possible collection of more accurate results. 


\section{Experimental Part}

This study was approved by Freiburg Ethics Committee (ref:014/1021), and conducted in accordance with the Declaration of Helsinki in Hamburg, Germany from January 2014 to February 2014.

\subsection{Volunteers}

Eight healthy volunteers of skin type I or II were recruited (6 female, 2 male). The mean age was $24.6 \pm 3.4(\mathrm{SD})$. All participants gave written informed consent before taking part in the study.

\subsection{Sunscreen}

Sunscreen (Eau thermale Avène SPF50+) was provided by Laboratoires Pierre Fabre (Toulouse, France). The active ingredients were bis-ethylhexyloxyphenol-methoxyphenyltriazine (Tinosorb-S), diethylhexyl-butamido-triazone (Uvasorb-HEB), butylmethoxydibenzoyl-methane (Parsol-1789), methylene-bis-benzotriazolyltetramethylbutylphenol (Tinosorb-M). The product also contained the antioxidant tocopherylglucoside. The SPF was $64.0 \pm 15.8$ (SD) and the in vitro UVA protection factor (UVA-PF) (ISO 24443 International Standard) was 25.3 with a critical wavelength of $379 \mathrm{~nm}$.

\subsection{Irradiation: UVR source:}

Simulated solar radiation (SSR) was obtained from a 300W Multiport system (SOLAR Light, Philadelphia, PA, USA), that was used with a UVR-transparent flexible light fiber bundle to irradiate the test areas with the requested irradiation intensity on spots with a diameter of approximately $1 \mathrm{~cm}$.

\subsection{Irradiation procedure and collection of the suction blisters}

At first, the MED of each subject was determined. The mean MED was 29.75 \pm 9.7 (SD) $\mathrm{mJ} / \mathrm{cm}^{2}$. For each volunteer, sunscreen was applied at $2 \mathrm{mg} / \mathrm{cm}^{2}$ on the interior of one forearm, 15-30 minutes before exposure to SSR. The other forearm was left untreated. On the sunscreenprotected arm, two zones were exposed to $15 \mathrm{MED}$. On the non-sunscreen-protected arm, two 
zones were exposed to $2 \mathrm{MED}$ and two skin zones were chosen as controls (non-SSR-exposed skin). The different skin zones were randomized among the volunteers.

After exposure to SSR, the sunscreen was removed. Then, six suction blisters of $6 \mathrm{~mm}$ were induced by applying a negative pressure to the skin zones (180 mbar during $30 \mathrm{~min}$ and 300 mbar afterwards) for approximately 4 hours. After the blisters were formed, the blister roofs, formed by the epidermis were carefully removed. Three blister roofs (non-exposed, 2MED exposed, photoprotected 15 MED exposed) were stored for HPLC-MS/MS analyses, and three analog samples were stored for CPD-immunostaining.

\subsection{HPLC-MS/MS analyses of pyrimidine dimers in suction blister's roofs}

Suction bilster's roofs were keep at $-80^{\circ} \mathrm{C}$ until use. For DNA extraction, the sample was first grinded by a metallic bead in a $2 \mathrm{ml}$ Eppendorf tubes containing ATL buffer (Qiagen) upon shaking in a Tissuelyzer apparatus (Qiagen). Proteinase K was added and the sample gently stirred at $55^{\circ} \mathrm{C}$ for $3 \mathrm{~h}$. Then, SDS was added and the sample was incubated for $30 \mathrm{~min}$ at $37^{\circ} \mathrm{C}$. A mixture of RNase A and T1 was added and incubation was resumed for $30 \mathrm{~min}$. A concentrated aqueous solution of sodium iodide was added and the sample vortexed. 2-Isopropanol was added and the sample was centrifuged. The supernatant was discarded and the DNA pellet made soluble in $50 \mu \mathrm{l}$ of deionized water. DNA was hydrolyzed by successive incubation with nuclease P1, DNase II and Phosphodiesterase II ( $\left.\mathrm{pH} 6,2 \mathrm{~h}, 37^{\circ} \mathrm{C}\right)$, and alkaline phosphatase and phosphodiesterase $\mathrm{I}\left(\mathrm{pH} 8,2 \mathrm{~h}, 37^{\circ} \mathrm{C}\right)$. The hydrolyzed samples were then analyzed by reverse phase HPLC. Detection involved first a UV detector which provided the amount of normal bases, and therefore the amount of analyzed DNA. The HPLC eluent was then directed toward an electrospray mass spectrometer operated in the multiple reaction mode. Using this specific and sensitive detection technique, the amounts of pyrimidine dimers was determined by external calibration using authentic standards. Results were expressed in number of dimer per million normal bases.

\subsection{Immunostaining of CPDs in suction blisters's roof and multi-photon imaging}

Suction skin blisters were fixed in $4 \%$ paraformaldehyde for 1 hour at room temperature (RT). Thereafter, suction blisters were permeabilized and blocked in 0,3\% Triton X100 / 1 \% BSA / PBS for 1 hour at RT under continuous agitation. For DNA denaturation, epidermal tissue were incubated with $2 \mathrm{~N} \mathrm{HCl}$ for $15 \mathrm{~min}$ at $\mathrm{RT}$. Then, suction blisters were washed in PBS and 
incubated with mouse anti-human CPDs antibody (1:1000, overnight, $4^{\circ} \mathrm{C}$; Cosmobio, Japan), and with Alexa-fluor® 568 Donkey anti-mouse secondary antibody (1:200, 1h, RT ; Invitrogen, France). Last, samples were washed in PBS and nuclei were stained with Hoechst (1:50 000, 30min, RT; Molecular Probes ${ }^{\mathrm{TM}}$, ThermoFischer, France). Slides were mounted using fluorescent mounting medium (Dako,USA).

Samples were imaged with a multiphoton microscope (A1 MP Si, Nikon) equipped with a tunable pulsed ultrafast laser (Laser MaiTai HP DeepSee, Spectra Physics), four non-descanned detectors (GaAsp NDD Nikon) and a filter cube (492nm, 525nm/50, 575nm/50, 629nm/53). An objective dedicated to MP microscopy was used (CFI75 Apochromat 25xW MP - NA 1.1 Nikon). For each sample, three stacks covering the entire depth were acquired at three different positions. Image processing was performed with Imaris software (Bitplane, Zürich, Switzerland). A very large number of nucleus was identified and segmented from the Hoechst staining in the blue channel. The CPD staining of each segmented nucleus was then evaluated in the red channel.

\subsection{Statistical analysis}

The comparison of the number of DNA damages between treatments was performed using a linear mixed model. In cases where residuals were not considered as normally distributed, DNA damages values were log-transformed. Side (left/right), position on the forearm (lower/upper) and treatment were taken as fixed factors. Subject was taken as random factor to take into account pairing between samples from the same subject. Pairwise comparisons of the treatments was made using differences of estimated means and p-value adjustment was performed using the Tukey method. The R software v3.2.3 (http://cran.r-project.org) was used for statistical analyses. The statistical significance level of the various 2 -sided tests was $5 \%$.

\section{Results}

\subsection{Quantification of photoproducts by HPLC-MS/MS}

In the present protocol, each volunteer provided 3 samples for HPLC-MS/MS analyses: 1 exposed to SSR without photoprotection, 1 exposed to SSR with photoprotection and 1 unexposed. For each suction blister, total DNA was extracted from the full $6 \mathrm{~mm}$ suction blister. 
DNA was then enzymatically hydrolyzed and analyzed by HPLC for the content in normal bases (UV detection) and dimeric photoproduct (MS/MS detection). The amount of DNA extracted from the blister's roof was quite satisfactory with a mean value of $4.3 \pm 1.8 \mu \mathrm{g}$ per sample. This sample size made possible the unambiguous detection of CPDs following exposure to 2 MED (Fig. 1). One exception was CC CPD that is the less frequent and was below the limit of detection. The ratio between the levels of CPDs was in line with data obtained on cultured cells or skin explants $[10,16,28,29]$. TT CPD was the main photoproduct, followed by TC and CT CPDs (Fig. 2). Another interesting feature is that of 64PPs and their Dewar valence isomers which result from the conversion of UVB-induced 64PPs by the UVA portion of the simulated sunlight spectrum [15,30] were detected only in minute amounts close to the detection limit in only a few samples of unprotected irradiated skin.

The photoproducts were not detected in non-exposed samples (Fig. 1), in agreement with the specific origin of pyrimidine dimers produced only by SSR. More interestingly, the amount of photoproduct was below the detection limit in samples exposed to SSR with a preliminary addition of sunscreen (Fig. 1). In the latter case a dose of 15 MED was used. The absence of detectable TT CPD in photoprotected samples shows the efficiency of the sunscreen. For statistical analyses, the level of CPD was set at half the limit of detection in unexposed and protected samples. ANOVA of the TT-CPD of all sites/assays/treatments showed a significant $\mathrm{p}$-value $(\mathrm{p}<0.001)$ and post hoc tests showing that the mean number of lesions on the nonphotoprotected skin was higher than both the non-exposed skin $(p<0.0001)$ and the photoprotected and exposed skin $(\mathrm{p}<0.0001)$.

\subsection{Visualization and semi-quantitative analysis of CPD by imaging}

Hoechst and CPD co-staining were performed on $1 \mathrm{~mm}$ punches taken from the suction blister. The blister's roof sample was directly stained and imaged without re-cutting it into slides. 3D images were acquired throughout the entire sample thickness in three different zones. All images were acquired with the same laser power and same detector gains. Nuclei were clearly identified on the blue channel by the Hoechst staining. On the red channel, CPD staining was very strong in the SSR-exposed and non-photoprotected samples, whereas it was very light in the SSR-exposed and photoprotected samples (Fig. 3). The non-exposed samples showed no CPD staining. Image analysis was performed with Imaris software. A very large number of nucleus (more than 400) was identified and segmented from the Hoechst staining in the blue 
channel. The CPD staining of each segmented nucleus was then evaluated in the red channel. Two analyses were performed on the segmented nuclei.

First of all, the mean CPD staining was computed from the mean red level of the cells. This procedure did not rely on any threshold definition. The SSR-exposed non-protected samples presented a very high mean red intensity compared to the non-SSR-exposed samples (Fig. 4a). The SSR-exposed and photoprotected samples presented a very low level of red fluorescence that was close to the intensity of the non-exposed samples. The red fluorescence intensity observed on the non-exposed samples was probably caused by non-specific background noise. ANOVA of the CPD staining of all sites showed a significant $\mathrm{p}$-value $(\mathrm{p}<0.001)$ and post hoc tests showed that the intensity of the unprotected skin was higher than the non-exposed $(\mathrm{p}<0.0001)$ and higher than the exposed photoprotected skin $(\mathrm{p}=0.0022)$.

The second approach aims at quantifying the number of CPD positive cells. Then, in order to correctly set a threshold for positive CPD staining, we analyzed all the non-exposed samples, as they should not contain any CPD positive cells. The threshold for considering positive staining was calculated from the non-exposed samples by considering that $98 \%$ of the cells were below the threshold. Low threshold values were determined for all donors. To process all the data exactly the same way, a unique threshold was established as the mean of subject thresholds. Criteria for positive staining having been established, the relative number of positive cells were then counted on each sample. The SSR-exposed unprotected samples presented $50.2 \%$ of positively CPD stained cells whereas it was only of $5.3 \%$ on the SSR-exposed photoprotected samples (Figure 4b)

ANOVA of the number of CPD positive cells of all sites showed a significant $p$-value $(p<0.001)$ and post hoc tests showed that the percentage of the not-photoprotected skin was higher than, both, the non-exposed skin $(\mathrm{p}<0.0001)$ and the photoprotected skin $(\mathrm{p}=0.0001)$. 


\section{Discussion}

This study addresses an important issue in the assessment of the photoprotection properties of sunscreens against the adverse effects of solar UVR in human skin, namely its prevention against carcinogenic properties of sunlight. Emphasis was placed on the formation of dimeric pyrimidine photoproducts in DNA which are at the origin of mutations leading to tumors. In order to prevent this deleterious process, DNA repair takes place in most skin cells afflicted with DNA lesions. Unfortunately, repair is not fully efficient and some cells can thus proliferate, accumulate mutations and become a precursor to skin cancer. A requisite in the understanding of the processes triggered by the UVR-induced genotoxic stress and in the evaluation of photoprotection strategies is the accurate quantification of DNA damage. Although cellular studies may provide valuable results, data are always needed in the whole tissue that may modulate the level of damage and where intracellular communication plays a major role in DNA damage response.

To our knowledge, all previous in-vivo studies related to link between formation of DNA photoproducts and sunscreens have been realized with skin biopsies. In the present work, we show that suction blister is an appropriate alternative sampling technique. This sampling procedure is much less invasive for the volunteers than biopsies since the dermal-epidermal junction is not affected. Therefore, only the epidermis is collected with the exception of its basal layer. Neither bleeding nor damage to dermis and hypodermis are observed. As a consequence, healing is fast (around 10 days) and does not leave scars. Suction blister is thus well suited for clinical studies requiring collection of multiple samples from a same donor without the ethical issues associated with biopsies. We therefore designed the present study to determine whether use of suction blisters was possible for the quantification of DNA photoproducts.

In our protocol, blisters of each donor were taken from skin either unexposed, exposed to SSR without sunscreen or exposed to SSR through sunscreen. Suction blister were collected, the roof was separated and DNA photoproducts were quantified. A first approach used was DNA extraction followed by HPLC-MS/MS measurement of individual dimeric photoproducts. In addition, through an accurate calibration, HPLC-MS/MS provides truly quantitative values [27]. This approach has been in used in human skin following ex-vivo exposure to UVR $[10,26,29]$, but rarely after in vivo irradiation. We found that suction blisters sampling was well suited for HPLC-MS/MS measurements, in particular in comparison with classical $3 \mathrm{~mm}$ 
biopsies that contain less cells. A few $\mu \mathrm{g}$ of DNA could be isolated It was thus possible to determine the photoproduct distribution. Access to larger amount of material than in small biopsies also allowed to work with low doses compatible with human experiments. The three major CPDs, TT TC and CT, were unambiguously detected in samples of skin exposed to 2 MED of SSR. In contrast, 64PPs and Dewars were absent from most DNA samples, far from the expected ratio between the levels of CPD and (64PP + Dewar) reported to range between 3 and 5 [8,28,31]. The reduced proportion of 64PPs and Dewars in the blisters' samples likely reflects repair taking place during the collection of the bubble. The bias induced by repair for the measurement of CPDs is expected to be much lower they are removed much more slowly [32-34].

The second technique applied to DNA damage measurement relied on immunohistochemistry, a commonly used method to measure skin photoproduct with specific antibodies targeted to CPDs or 64PPs [35]. This technique is usually considered only as semi-quantitative, because the measured fluorescence intensity cannot be directly expressed as a precise number of DNA lesions. Yet, it provides valuable information on the localization of the DNA damage in the tissue. We thus optimized the technique for suction blisters. First, we developed an approach making possible the direct staining on floating roof samples. Then, we performed 3D imaging with multi-photon microscopy, an effective method for 3D imaging of thick tissues. This novel procedure enabled us to analyze a much larger number of cells than the usual analyses performed with transverse slides. Moreover, 3D microscopy coupled with image processing enabled us to evaluate the mean staining intensity of individual cells. The epidermis was easily imaged at all layers. No clear staining gradient was observed through the sample's thickness. This observation shows that UVR reaches all epidermal layers, and that basal layer cells are also exposed to UVR. The percentage of cells that present DNA lesions after a solar UV exposure was then computed, and it was shown that $50 \%$ of epidermal cells present photoproducts with 2 MED exposure.

The methodological developments described above were then applied to the evaluation of photoprotection in skin. The existing international norms on sunscreen protection factor (SPF) evaluation are based on in vitro measurements and the in-vivo SPF method [3]. The latter is drawn from erythema evaluation which translates a global inflammatory skin response. This biological end-point is not directly relevant to one of the most adverse effect of UVR on skin that is cancer. However, sunscreens strongly reduce the UVR dose that reaches the nucleus of skin cells, prevent the formation of DNA photoproducts and thereby reduce the risk of skin 
cancer. In that respect, the approaches developed in the present work for the quantification of DNA lesions may permit sunscreen manufacturers to more accurately gauge the efficacy of their products. Using HPLC-MS/MS, we observed for all subjects that the level of all dimeric photoproducts was strongly reduced upon exposure of skin protected by a sunscreen. The amount of DNA photoproducts, including the mutagenic cytosine-containing CPDs, were below the detection limit. Similar conclusions were drawn by using immunostaining. The labeling was back to basal level when skin was exposed to SSR in the presence of sunscreen. It may thus be concluded, in agreement with other works [20,21,24,36], that sunscreens efficiently protect skin DNA.

In summary, the present study brought to light two points of great interest. First, at a technical level, we have combined the suction blister sampling technique with accurate HPLC-MS/MS measurements and with multi-photon microscopy, allowing better immuno-staining quantification of photoproducts. Secondly, from a public health perspective, we have shown that the sunscreens such as the SPF 50+ presently used provide a very high protection against DNA photoproduct formation in the skin. We thus believe that this approach deserves to be more widely considered. Future developments will include increasing the sensitivity of the assays in order to detect residual DNA damage in protected skin and determine an actual DNA protection factor. Measurements in the DNA suction blister could be also extended to other damage such as oxidized bases resulting from SSR-induced oxidative stress but also those produced by chemicals and pollutants.

\section{5 .References}

[1] F. El Ghissassi, R. Baan, K. Straif, Y. Grosse, B. Secretan, V. Bouvard, L. BenbrahimTallaa, N. Guha, C. Freeman, L. Galichet, V. Cogliano, A review of human carcinogensPart D: radiation, Lancet Oncol. 10 (2009) 751-752.

[2] V.O. Melnikova, H.N. Ananthaswamy, Cellular and molecular events leading to the development of skin cancer, Mutat. Res. 571 (2005) 91-106.

[3] C.S. COLIPA, JCIA, CTFA, International sun protection factor (SPF) test method, COLIPA Guideline (2006)

[4] D. Moyal, M. Pissavini, F. Boyer, P. V., J.H. Frelon, In vivo persistent pigment darkening method: proposal of a new standard product for UVA protection factor determination, Int. J. Cosmet. Sci. 29 (2007) 443-449.

[5] J. Cadet, S. Mouret, J.L. Ravanat, T. Douki, Photoinduced damage to cellular DNA: direct and photosensitized reactions, Photochem. Photobiol. 88 (2012) 1048-65. 
[6] D.E. Brash, J.A. Rudolph, J.A. Simon, A. Lin, G.J. McKenna, H.P. Baden, A.J. Halperin, J. Ponten, A role for sunlight in skin cancer: UV-induced p53 mutations in squamous cell carcinoma, Proc. Natl. Acad. Sci. USA 88 (1991) 10124-10128.

[7] J. Cadet, T. Douki, J.L. Ravanat, P. Di Mascio, Sensitized formation of oxidatively generated damage to cellular DNA by UVA radiation, Photochem. Photobiol. Sci. 8 (2009) 903-11.

[8] D. Perdiz, P. Grof, M. Mezzina, O. Nikaido, E. Moustacchi, E. Sage, Distribution and repair of bipyrimidine photoproducts in solar UV-irradiated mammalian cells. Possible role of Dewar photoproducts in solar mutagenesis, J. Biol. Chem. 275 (2000) 2673226742.

[9] A.R. Young, C.S. Potten, O. Nikaido, P.G. Parsons, J. Boenders, J.M. Ramsden, C.A. Chadmick, Human melanocytes and keratinocytes exposed to UVB or UVA in vivo show comparable levels of thymine dimers, J. Invest. Dermatol. 111 (1998) 936-940.

[10] S. Mouret, C. Baudouin, M. Charveron, A. Favier, J. Cadet, T. Douki, Cyclobutane pyrimidine dimers are predominant DNA lesions in whole human skin exposed to UVA radiation, Proc. Natl. Acad. Sci. USA 103 (2006) 13765-13770.

[11] S. Mouret, C. Philippe, J. Gracia-Chantegrel, A. Banyasz, S. Karpati, D. Markovitsi, T. Douki UVA-induced cyclobutane pyrimidine dimers in DNA: a direct photochemical mechanism?, Org. Biomolec. Chem. 8 (2010) 1706-1711.

[12] B. Cortat, C.C.M. Garcia, A. Quinet, A.P. Schuch, K.M. de Lima-Bessa, C.F.M. Menck, The relative roles of DNA damage induced by UVA irradiation in human cells, Photochemical \& Photobiological Sciences 12 (2013) 1483-1495.

[13] A.P. Schuch, R.D. Galhardo, K.M. de Lima-Bessa, N.J. Schuch, C.F.M. Menck, Development of a DNA-dosimeter system for monitoring the effects of solar-ultraviolet radiation, Photochemical \& Photobiological Sciences 8 (2009) 111-120.

[14] T. Douki, A. Reynaud-Angelin, J. Cadet, E. Sage, Bipyrimidine photoproducts rather than oxidative lesions are the main type of DNA damage involved in the genotoxic effect of solar UVA radiation, Biochemistry 42 (2003) 9221-9226.

[15] T. Douki, E. Sage, Dewar valence isomers, the third type of environmentally relevant DNA photoproducts induced by solar radiation, Photochem. Photobiol. Sci. 15 (2015) 2430.

[16] S. Courdavault, C. Baudouin, M. Charveron, A. Favier, J. Cadet, T. Douki, Larger yield of cyclobutane dimers than 8 oxo-7,8-dihydroguanine in the DNA of UVA-irradiated human skin cells, Mutat. Res. 556 (2004) 135-142.

[17] M. Al Mahroos, M. Yaar, T.J. Phillips, J. Bhawan, B.A. Gilchrest, Effect of sunscreen application on UV-induced thymine dimers, Arch. Dermatol. 138 (2002) 1480-1485.

[18] S. Arase, E.G. Jung, In vitro evaluation of the photoprotective efficacy of sunscreens against DNA damage by UVB, Photodermatology 3 (1986) 56-59.

[19] D. Bacqueville, A. Mavon, Comparative analysis of solar radiation-induced cellular damage between ex vivo porcine skin organ culture and in vitro reconstructed human epidermis, Int. J. Cosmet. Sci. 31 (2009) 293-302.

[20] V. Bissonauth, R. Drouin, D.L. Mitchell, M. Rhainds, J. Claveau, M. Rouabhia, The efficacy of a broad-spectrum sunscreen to protect engineered human skin from tissue and DNA damage induced by solar ultraviolet exposure, Clinic. Cancer Res. 6 (2000) 41284135.

[21] S.E. Freeman, R.D. Ley, K.D. Ley, Sunscreen protection against UV-induced pyrimidine dimers in DNA of human skin in situ, Photodermatology 5 (1988) 243-247.

[22] M. Heenen, P.U. Giacomoni, P. Golstein, Individual variations in the correlation between erythemal threshold, UV-induced DNA damage and sun-burn cell formation, J. Photochem. Photobiol. B:Biol. 63 (2001) 84-87. 
[23] M.C. van Praag, L. Roza, B.W. Boom, C. Out-Luijting, J.B. Henegouwen, B.J. Vermeer, A.M. Mommaas, Determination of the photoprotective efficacy of a topical sunscreen against UVB-induced DNA damage in human epidermis, J. Photochem. Photobiol. B:Biol. 19 (1993) 129-134.

[24] A.R. Young, J.M. Sheehan, C.A. Chadwick, C.S. Potten, Protection by ultraviolet A and B sunscreens against in situ dipyrimidine photolesions in human epidermis is comparable to protection against sunburn, J. Invest. Dermatol. 115 (2000) 37-41.

[25] D. Bacqueville, T. Douki, L. Duprat, S. Rebelo-Moreira, B. Guiraud, H. Dromigny, V. Perier, S. Bessou-Touya, H. Duplan, A new hair follicle-derived human epidermal model for the evaluation of sunscreen genoprotection, J. Photochem. Photobiol. B:Biol. 151 (2015) 31-38.

[26] S. Mouret, P. Bogdanowicz, M.J. Haure, N. Castex-Rizzi, J. Cadet, A. Favier, T. Douki, Assessment of the photoprotection properties of sunscreens by chromatographic measurement of DNA damage in skin explants, Photochem. Photobiol. 87 (2011) 109-16.

[27] T. Douki, The variety of UV-induced pyrimidine dimeric photoproducts in DNA as shown by chromatographic quantification methods, Photochem. Photobiol. Sci. 12 (2013) 12861302.

[28] T. Douki, J. Cadet, Individual determination of the yield of the main-UV induced dimeric pyrimidine photoproducts in DNA suggests a high mutagenicity of CC photolesions, Biochemistry 40 (2001) 2495-2501.

[29] S. Mouret, M.T. Leccia, J.L. Bourrain, T. Douki, J.C. Beani, Individual photosensitivity of human skin and UVA-induced pyrimidine dimers in DNA, J Invest Dermatol 131 (2011) 1539-46.

[30] T. Douki, Relative Contributions of UVB and UVA to the Photoconversion of (6-4) Photoproducts into their Dewar Valence Isomers, Photochem. Photobiol. 92 (2016) 58794.

[31] D.L. Mitchell, J.P. Allison, R.S. Nairn, Immunoprecipitation of pyrimidine(64)pyrimidone photoproducts and cyclobutane pyrimidine dimers in UV-irradiated DNA, Radiat. Res. 123 (1990) 299-303.

[32] D.L. Mitchell, C.A. Haipek, J.M. Clarkson, (6-4) Photoproducts are removed from the DNA of UV-irradiated mammalian cells more efficiently than cyclobutane pyrimidine dimers, Mutat. Res. 143 (1985) 109-112.

[33] S. Mouret, M. Charveron, A. Favier, J. Cadet, T. Douki, Differential repair of UVBinduced cyclobutane pyrimidine dimers in cultured human skin cells and whole human skin, DNA Repair 7 (2008) 704-712.

[34] A.R. Young, C.A. Chadwick, G.I. Harrison, J.L. Hawk, O. Nikaido, C.S. Potten, The in situ repair kinetics of epidermal thymine dimers and 6-4 photoproducts in human skin types I and II, J. Invest. Dermatol. 106 (1996) 1307-1313.

[35] T.R. Berton, D.L. Mitchell, Quantification of DNA photoproducts in mammalian cell DNA using radioimmunoassay, Meth. Molec. Biol. 920 (2012) 177-87.

[36] M.C. van Praag, L. Roza, B.W. Boom, C. Out-Luijting, J.B. Henegouwen, B.J. Vermeer, A.M. Mommaas, Determination of the photoprotective efficacy of a topical sunscreen against UVB-induced DNA damage in human epidermis, J. Photochem. Photobiol. B:Biol. 19 (1993) 129-134. 


\section{Legends to Figures}

Figure 1: Formation of CPDs in human skin exposed to 2 MED measured in roofs of suction bubbles. The figure show representative HPLC-MS/MS chromatogram obtained during analysis of DNA extracted from a) non-exposed skin, b) unprotected skin exposed to 2 MED and c) skin protected by $2 \mathrm{mg} / \mathrm{cm}^{2}$ of SPF50+ sunscreen and exposed to $15 \mathrm{MED}$.

Figure 2: Distribution of TT, TC and CT CPDs in the DNA of skin exposed to 2 MED SSR without photoprotection. Reported values are expressed in number of CPDs per millions bases and are means \pm standard error $(n=8)$.

Figure 3: a) 3D volumes of suction blister roofs staining and b) Maximum Intensity Projections, Hoechst staining (blue channel), CPD staining (red channel): non-exposed skin (NE), 2 MED exposed skin (E) and 15 MED exposed sunscreen photo-protected skin (EP).

Figure 4: a) Intensity of nuclei CPD staining (red channel) and b) percentage of CPD positive nuclei for non-exposed skin (NE), 2 MED exposed skin (E) and 15MED exposed sunscreen photo-protected skin (EP). Reported values are means \pm standard error $(n=8)$. 


\section{Figure 1}
a) Non-exposed skin
b) Non-protected and exposed (2 MED)
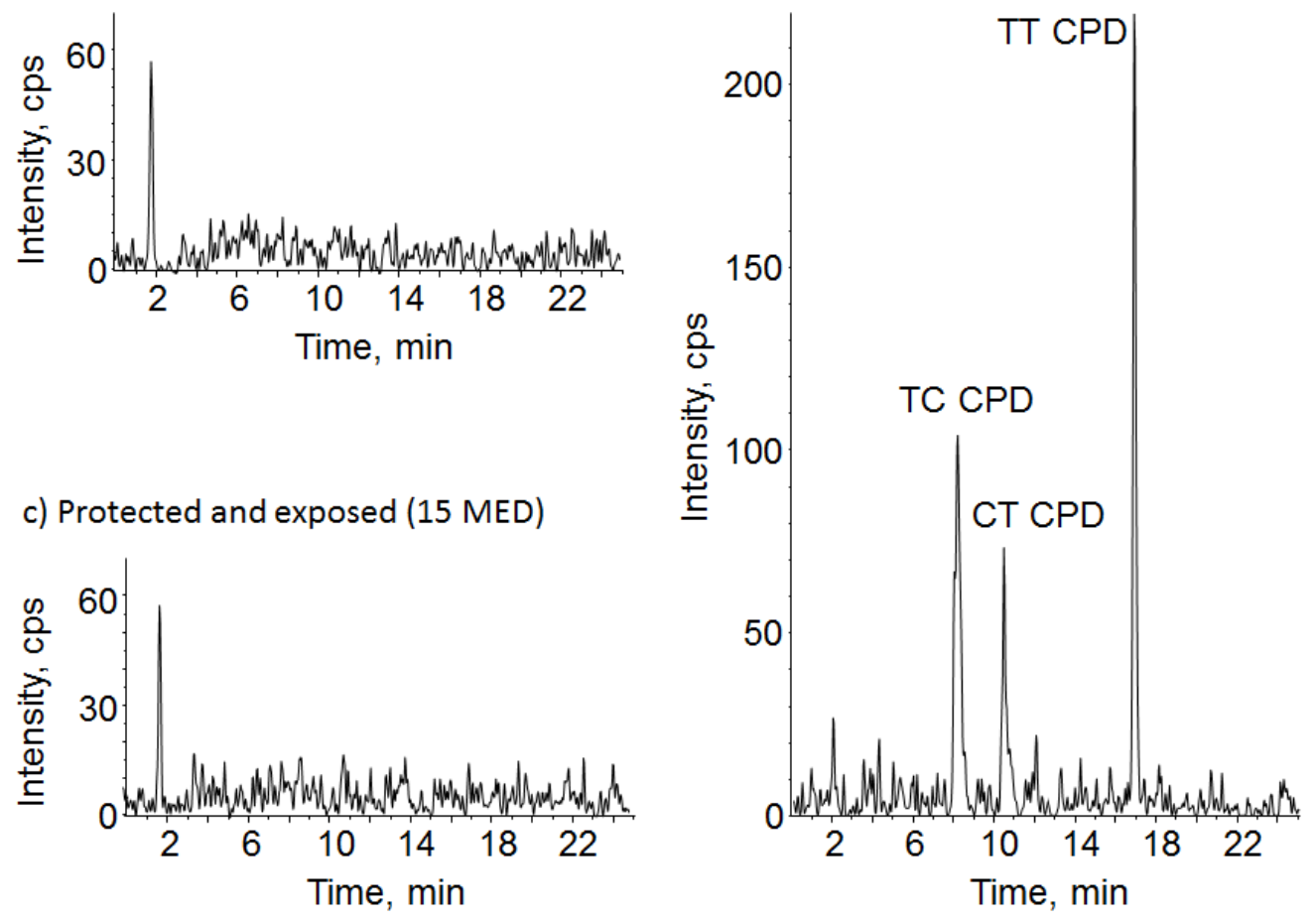

Figure 2

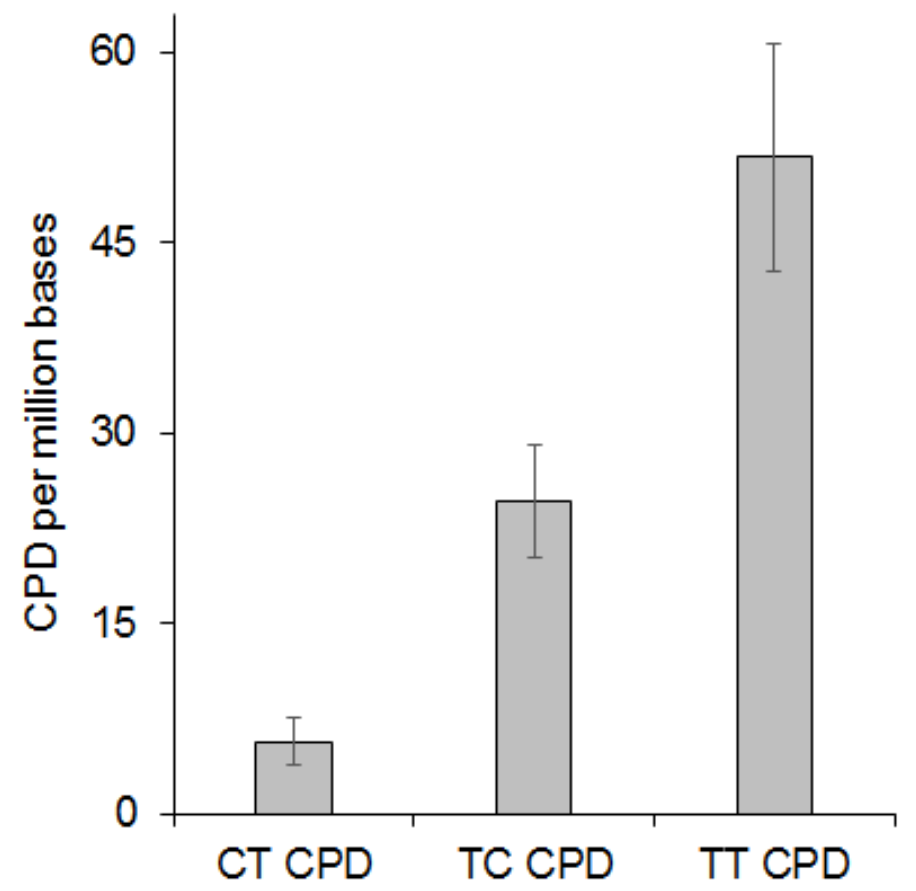


Figure 3:

Figure 4: 